\title{
LA ARgumENTACIÓN JuRÍdicA COMO MÉTODO CIENTÍFICO DE VERIFICACIÓN ${ }^{1}$
}

Gonzalo LeVi OBREgón SALinas ${ }^{2}$

RESUMEN: La presente investigación busca utilizar a la Teoría de la Argumentación Jurídica, como método de verificación de la ciencia jurídica mediante las variables: Verdad, Lenguaje, Teoría, Tiempo y Consciencia.

Palabras clave: Argumentación jurídica, ciencia jurídica, Verdad, Lenguaje, Teoría, Tiempo y Consciencia.

ABSTRACT: The present research seeks to use the Theory of Legal Argumentation, as a method of verification of legal science through the elements: Truth, Language, Theory, Time and Consciousness.

Key Words: Legal Argumentation, legal science, Truth, Language, Theory, Time and Consciousness.

SumARIO: I. El contexto de los paradigmas jurídicos en México., II. La Teoría de la Argumentación Jurídica como método de verificación., III. Los elementos de la Teoría de la Argumentación Jurídica, como método científico de verificación dentro de la ciencia jurídica, 1. La verdad., 2. Lenguaje, 3. Teoría, 4. Tiempo, 5. Consciencia, IV. Bibliografía.

\section{El contexto de los paradigmas jurídicos en México}

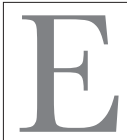

n la práctica del derecho en México, así como su estudio ha sido necesario que los operadores reformulen la manera de pensar, argumentar el derecho, la tradición positivista en

1 El presente trabajo es realizado bajo el apoyo obtenido por parte del CONACYT, en el marco de la estancia posdoctoral 2018 (1).

2 Doctor en Derecho por el programa de posgrado en Derecho de la UNAM, profesor de la materia Argumentación Jurídica, y Sistemas de Control Constitucional, en el programa de posgrado en Derecho de la UNAM, Facultad de Estudios Superiores Acatlán, <gonzaloabogado@outlook.com>. 
nuestra Estado-Nación, aun continúa vigente y oponiéndose al cambio de paradigma desde la perspectiva de la ciencia jurídica, tener que reformular el planteamiento de que solamente resolver con lo que se encuentra en la legislación o de un sistema normativo.

Después de tantos años de ser el formalismo jurídico la única propuesta no por la ciencia jurídica sino por el sistema jurídico mexicano, los juzgadores, así como los practicantes tienen la postura de basarse únicamente al supuesto normativo, revisemos la ley para saber cuál es la aplicación que tendrá, como si no fuera necesario pensar o realizar una abstracción de la situación, la conclusión que ofrece la norma jurídica es innecesario el proceso de análisis en cuanto al supuesto.

Las conclusiones que ofrece la norma jurídica pueden beneficiar a una visión positivista desde no realizar un cuestionamiento sobre la existencia de la norma jurídica no desde el aspecto legislativo porque entendemos que la formalidad de legislación es lo que lo hace obligatoria desde el aspecto formal de la norma jurídica, por ello, basta tener como premisa lo que la norma jurídica ya dice de manera concreta, y aceptar que sus conclusiones como correctas y verdaderas.

Esto implica que en el positivismo normativo hay que partir de que la norma jurídica es verdad desde varias perspectivas la primera es considerar que la norma jurídica regula de manera correcta lo que el hecho establece, y dos que las consecuencias jurídicas previstas son las necesarias no sólo de la regulación dentro del sistema jurídico, sino también desde el contexto de los hechos relacionados con los sucesos del día a día de las personas y sus características.

"Dentro de la práctica jurídica, así como en el estudio del Derecho, nos encontramos con que el el Estado Legislativo de Derecho era la manera de pensar, crear y resolver los acontecimientos que al Derecho correspondiera resolver, con el único fundamento de la 
búsqueda del formalismo jurídico como la preeminencia al momento de aplicar la ley sobre cualquier situación o persona."3

La falta de cuestionamiento del positivismo normativo por parte de los operadores favorece la creación de normas jurídicas que no respeten las características de las personas, no exista razón dentro de su contenido además de alentar la existencia de contradicciones o colisiones incluso entre la legislación, ya sea federal, estatal o local, de igual manera considerar que a falta de regulación expresa y como último recurso se aplicaran los principios generales del derecho, ante la falta de regulación concreta de un supuesto normativo.

Lo que permite que el sistema jurídico sea lógico desde la perspectiva de la formalidad debido a que cumple los procedimientos para que sea llamada legislación o norma vigente y por ello valida, en este contexto del paradigma positivista es el que México ha encontrado una aplicación teórica y se ha permitido durante mucho tiempo la aplicación únicamente de la ley, desde su perspectiva formalista, para lo cual es creada, con ello, el principio de legalidad permanece intocado.

La reflexión sobre este aspecto de las cosas revela una verdad sensata: el paso desde las formas simples de la sociedad, donde las reglas primarias de oblgiación son el único medio de control social, al mundo jurídico, con su legislatura centralmente organizada, sus tribunales, funcionarios y sanciones, trae consigo ventajas considerables, pero a un cierto precio. El precio es el riesgo de que el poder centralmente organizado puede ser empleado para la opresión de muchos, cuyo apoyo no le resulta imprescindible, de una manera que el régimen más simple de reglas primarias no podía llevar a cabo. ${ }^{4}$

El cuestionamiento básico que tendríamos que realizar sería, ¿la norma jurídica con base en los formalismos y sus instituciones nos

3 Gonzalo Levi, Obregón Salinas., Lo Teórico y lo Practico de los Derechos Humanos., Ciudad de México, Thomson Reuters, 2018, p. 76.

4 H. L. A. Hart., El concepto de Derecho., Buenos Aires., AbeledoPerrot., 2012, pp. $249-250$. 
lleva a la verdad?, es decir el positivismo jurídico aplicado en México ¿conduce a la norma jurídica a que identifique los supuestos normativos en su regulación?, ¿existe una etapa dentro del proceso legislativo para observar la necesidad de regulación de una norma jurídica?, la norma jurídica y la verdad, ¿serán aspectos que conforman una solución ambivalente a las necesidades de las personas?

Desde la perspectiva de la verdad en la ciencia jurídica podríamos considerar como verdadero la perspectiva del acontecimiento en relación con lo que la norma jurídica dice, tomando como premisa las consecuencias relacionadas con el acontecimiento, es decir desde la facticidad acontecida, o bien la necesidad reflejada en el actuar de las personas, partiendo de eso, más aun, de su comportamiento actual, tomando como generación, de la variable tiempo, en que la sociedad se desenvuelve.

"Pero si la verdad es una construcción, entonces las formas de presentarse la verdad será la construcción de un discurso que se sustenta en la concatenación lógica, racional, razonable y lingüística de las diferentes narraciones (narración en primera persona desde las testimoniales, narraciones explicativas desde los informes médicos y las periciales, etc.) que obran en el expediente"5

¿Cómo identificar cuando la disposición normativa ya no regula la verdad?, los acontecimientos relacionados dentro de la sociedad son un parámetro de confrontación a las disposiciones normativas o leyes, lo que podría ser muy sencillo de identificar la problemática consiste en que los cambios sociales deberían ser regulados inmediatamente pero quién decide cuándo regularlos, hasta cuándo una norma jurídica debería modificarse, para ello, es necesario que el legislador así lo decida, en que momento el legislador sabrá que tiene que existir el cambio de supuesto normativo.

De lo anterior podríamos llegar al primer cuestionamiento, ¿cuanto tiempo tiene que esperar la persona para que se le reconoz-

5 Gerardo, Ribeiro Toral., Verdady Argumentación furídica., Guanajuato, Porrúa, 2009, p. 29. 
ca un derecho mediante un supuesto normativo?, tenemos que esperar a que sea el legislador que decida el momento para modificar la vida de las personas, en qué momento para estas personas podría dejar de ser verdadero lo que la norma jurídica dice en relación a la regulación del hecho pero más aun, a las consecuencias que existen ante la falta de actualización de la norma jurídica.

La actualización de la norma jurídica debe ser desde la perspectiva directa e inmediata de la necesidad de las personas debido a que comprende su dignidad humana, la problemática de el Estado Legislativo se presenta con la actualización y aplicación de la norma jurídica, tiene que ser desde la petición de el gobernado ya que es de manera inmediata ante una autoridad como lo es el juzgador.

La problemática a resolver por el juzgador es que el gobernado le encomiende su petición de actualizar su necesidad frente a la norma jurídica obsoleta, el resolver o no por parte del juzgador dependerá de el paradigma que aplique el juzgador, debido a que si su estudio, abstracción, verificación tiene como contexto el Estado Legislativo, aplicará una disposición normativa que lejos de ayudarle le creará una nueva transgresión derivado de la aplicación normativa que no tiene actualización.

¿Cuál sería la diferencia de aplicar un paradigma o resolver el planteamiento con otro?, tiene que ver con la realidad de la resolución, con la intensión de proteger la dignidad humana o cualquier otro derecho humano, debido a que en el paradigma del Estado Legislativo se encuentra condicionada la creación exacta, así como aplicación idéntica del supuesto normativo frente a la dignidad humana o derecho humano, que durante el positivismo vigente como postura científica solamente utilizará lo que la norma jurídica diga, dejando de lado la falta de regulación actualizada de la norma jurídica.

En lo sucesivo podemos mantener en mente la posibilidad de que la Jurisprudencia sea interpretada tecnológicamente. Estoy consciente de que la palabra tecnología no resulta especialmente querida dentro del contexto social y ello en virtud de que muchas veces se asocia - de ma- 
nera toalmente errónea y aribtraria- con el término "tecnocracia". Quizás si se emplea el concepto de "arte" - lo que apunta eventualmente a lo mismo - entonces la cuestión se torna más aceptable. Pues se podría tener en mente una interpteación de la jurisprudencia en el sentido de una tecnlogía social. Es decir de un "arte" que sirva para preparar la toma de decisiones de cierto tipo. ${ }^{6}$

Esa es la problemática científica de la tecnología denominada norma jurídica, necesita una actualización constante por parte del poder legislativo, que se compone por ideologías políticas, y diferentes procedimientos para hacer cumplir la dignidad humana, de las personas, por lo que la falta de actualización de la norma jurídica como tecnología genera la complejidad para hacer aplicables los derechos humanos de los gobernados o seres humanos, con ello, no basta identificar la problemática dentro de un paradigma o la aplicación de la ley, hay que dar solución a los problemas que se presentan.

"De ser así, entonces los enunciados sobre la vigencia fáctica de las normas no serían otra cosa más que enunciados sobre hechos sociales, a partir de los cuales sería imposible derivar consecuencias normativas..."7

La complejidad del Estado Legislativo, va relacionado con la creación de los términos jurídicos que se implementan lo que hace imposible que las personas tengan un acercamiento con sus derechos que son reconocidos en la ley, debido a que a las personas que están destinadas las normas no entenderán no sólo de el contenido de la norma jurídica, más aun, de las consecuencias que tiene su comportamiento, la complejidad en la creación de los conceptos del derecho hace que cada vez más se aleje la intensión de su función.

Se entiende que la norma jurídica respeta a las disposiciones jurídicas jerarquicamente superiores a ésta, por ello cuando se

6 Hans, Albert., La ciencia del derecho como ciencia real., Ciudad de México, Fontamara, 2007, p. 77.

7 Hans, Albert., op cit., p. 79. 
emite una disposición normativa de manera general, en apariencia la norma secundaria, se debe apegar a un principio positivado en una norma superior, pero sin justificación la legislación secundaria se aparta de la legislación formalmente superior, y hace evidente una excepción no sólo a la norma jurídica que regula el principio además crea una regla de inaplicación de una norma con mayor jerarquía. Problemáticas formalistas que presenta el Estado Legislativo en la actualidad debido a la justificación de validez derivado del proceso de creación de las disposiciones noramtivas.

\section{La Teoría de la Argumentación Jurídica como método DE VERIFICACIÓN}

La problemática que nos encontramos en México, no sólo es el reto de la complejidad normativa, también es el contexto del cambio de paradigma en su aplicación, no basta con determinar o identificar que existe una nueva o diferente forma de resolver o dar solución a los planteamientos jurídicos, supuestos, contradicciones, falta de regulación jurídica, hay que resolver con base en el nuevo paradigma, así como identificar porque es la mejor manera de no sólo desde la perspectiva de la necesidad de la sociedad, además por la mejor funcionalidad de científica.

¿Cómo identificar en el campo de la ciencia jurídica la modificación de un paradigma?, así como la mejor solución a los problemas que se presentan, en el caso de México se percibe cuando lo esperado por la norma jurídica es distinto a lo que sucede en la vida de las personas, por ello, tomaremos como primer elemento de la teoría de la argumentación jurídica la verdad, consideraremos como verdad, los resultados que son independiente a la norma jurídica, lo que pasa en la vida de las personas y sus derechos humanos independiente a lo que la norma jurídica dice.

"Aristóteles insiste en que las premisas de una explicación científica deben ser verdaderas; claramente señala: 'El conocimiento de- 
mostrativo debe descansar en verdades fundamentales necesarias'. La exigencia de que las premisas sean verdaderas es uno de los requerimientos extralógicos que Aristóteles impone en las premisas de la demostración científica."8

La verdad puede ser vista dentro de esta teoría del Estado Constitucional, como el derecho humano que tiene el gobernado a la verdad, o bien el resultado de la contraposición o verificación de lo que dice la norma jurídica y se espera como resultado en la vida de las personas, frente a lo que pasa en su día a día, con ello, la verdad como elemento de la Teoría de la Argumentación Jurídica como elemento científico de verificación de la verdad tanto en el comportamiento de las autoridades, como el desenvolvimiento normativo en la regulación de los supuestos fácticos.

¿Existe la necesidad de buscar la verdad dentro de un Paradigma en la ciencia jurídica?, ¿por qué sería necesario identificar la verdad no sólo en la norma jurídica también en sus resultados o consecuencias?, la necesidad consideramos existe al encontrarse la aplicación de dos paradigmas en un mismo sistema jurídico, por un lado se encuentran las autoridades, abogados, doctrinarios que se aferran a la aplicación de su visión científica.

Por el otro lado, están los operadores que se encuentran identificados con la aplicación de los derechos humanos ya sea juzgadores, abogados, académicos, que han visto la necesidad en un cambio dentro de la ciencia jurídica, que impacte en la sociedad debido a los retos para resolver sus problemáticas así como el de las personas para llegar al bien común, con ello, los partidarios del Estado Constitucional, buscan la aplicación de la ciencia jurídica con la mayor inserción con todas las tecnologías posibles, dentro de su aspecto científico, no sólo tomar a la norma jurídica y como referente dentro de la ciencia jurídica.

8 Rolando, Tamayo y Salmorán., El derecho y la ciencia del derecho, Introducción a la ciencia jurídica, Ciudad de México, fontamara, 2011, p. 115. 
"El científico debe inducir principios y deducir de ellos enunciados sobre los fenómenos. Para Aristóteles el conocimiento científico comienza con el conocimiento de que cierto evento ocurre o de que ciertas propiedades coexisten. Sin embargo la verdadera explicación científica se logra únicamente cuando enunciados sobre esos eventos o propiedades son deducidos de los principios explicativos. La explicación científica es una transición del conocimiento de los hechos al conocimientos de las causas de los hechos."9

El Estado Constitucional tiene como característica insertar la verdad tanto como derecho humano, así como dentro de la ciencia jurídica, por ello, se parte de la ciencia debido a que no encontraremos en el valor, moralidad, ideología política la aplicación objetiva de la norma jurídica, así como de alguna teoría o postura, tendremos que recurrir a la ciencia en general para buscar el elemento de verificación es decir que tanto los conceptos puedan identificarse con su significado, frente a las consecuencias o resultados esperados.

No buscar solamente el valor que una sociedad impone, más aun, en México como nación existen distintas sociedades que se pueden evidenciar desde la desigualdad económica, o de la educación, también desde las características religiosas que moralmente puedan dar a la sociedad, lo que hace que cada persona de manera interna o individual tenga su propia consideración de lo que es un valor o moralidad, por ello, la ciencia jurídica debe estar en constante búsqueda de la verdad, tanto en la norma jurídica como sus conclusiones enfocadas a los derechos humanos.

La variable o concepto de verdad que conforma la teoría de la argumentación jurídica como método científico de verificación tiene como objeto excluir a las posturas subjetivas que solamente tengan el sustento ya sea formalista, es decir, que debido a la regulación en un cuerpo normativo sea justificación para su aplicación y modificar la vida de las personas o bien la posibilidad de poder

9 Rolando, Tamayo y Salmorán., op cit., p. 112. 
definir los supuestos normativos, posturas, ó límites a los derechos humanos de las personas, sin tener un referente objetivo.

Con ello, se retoma en el Estado Constitucional la posibilidad de aplicar científicamente el elemento de verdad para su verificación dentro de una Teoría como lo es la argumentación jurídica, en el marco del Estado Constitucional que tiene la característica de incluir a la verdad como derecho humano, y fin dentro de la ciencia jurídica para realizar la verificación tanto de las regulaciones de los derechos humanos en la ley como de los resultados que tiene la legislación en su dignidad y entorno.

El Estado Constitucional presupone que las personas, es decir, los ciudadanos, tienen un interés en la "búsqueda de la verdad"; donde el interés por la verdad es ya la meta a alcanzar. Incluso tiene el permitido el obligar - como ultima ratio - bajo juramento a cumplir conforme a la verdad subjetiva, aunque esto no quiere decir que garantice la verdad objetiva. El Estado puede encomendar a sus funcionarios (los de tercer poder por ejemplo) la búsqueda de la verdad también crear comisiones paralamentarias o "comités particulares", Pero "ministerios de la verdad" quedan estrictamente prohibidos. ${ }^{10}$

Por ello, es tomado en cuenta la verdad como elemento o variable dentro de la argumentación jurídica, más aun, que también es un derecho humano, por ello, deben ser aplicados desde la perspectiva de la ciencia jurídica, las tecnologías, o directrices que permitan verificar tanto la norma jurídica (considerada también sólo una tecnología de la ciencia), como sus consecuencias para abatir la problemática de lo dúctil que puede ser la norma jurídica, al momento de su creación por parte del poder legislativo, que no existe ningún procedimiento para identificar la creación de la norma jurídica, en relación a las necesidades de los gobernados que se tome en cuenta la verdad como referente.

10 Peter, Häberle., Verdad y Estado Constitucional., Ciudad de México, UNAMIIJ, 2006, p. 134. 
La importancia de la verdad dentro de la ciencia jurídica es para identificar las diferencias entre los paradigmas y no confundirlos en cuanto a la aplicación de los derechos humanos, entender que los derechos humanos se rigen por otro tipo de requisitos o principios científicos como la universalidad, progresividad, indivisibilidad, e interdependencia, por lo que esperar a que el legislador pretende definirlos así como identificar uno por uno a los derechos humanos en las normas jurídicas además de ser obsoleto desde la ciencia jurídica, es contradictorio a estos.

"Extremando los términos se puede preguntar si el orden jurídico incluyendo a la Constitución, puede descansar en la "mentira". De alguna manera es legítima la pregunta porque el derecho, como producto humano, puede descansar en el error, sobre todo en el caso de Constituciones inspiradas en el derecho positivo, ya que la positividad siempre puede ser cuestionada... Sin duda alguna hay Constituciones que no tienen vínculo alguno con la realidad." "

La contradicción consiste en que la regulación específica así como identificable genera limitaciones trascendentales ya que se le podría llamar de otra forma o bien reducirlo a otro tipo de derechos, más aun, pretender que para su acceso son necesarias garantías, lo cual en el derecho humano, la garantía en todo caso es el actuar de la autoridad, por ello, no se necesitan garantías que los protejan, por la obligación de la autoridad de garantizarlos desde su competencia.

En un momento histórico como lo es el cambio de paradigma del Estado Legislativo al Constitucional, hoy más que nunca se necesita identificar cuáles son las posturas y visiones que han sido modificadas, para evitar y sobreponerse a la reducción de los derechos humanos, o que se pretendan insertar en un paradigma anterior, lo que tendríamos como resultado la inaplicación de estos, así como un retroceso científico de la protección de las personas.

11 Peter, Häberle., op cit., pp. 139 -140. 
"Una explicación de la problemática de la falta de validez sustancial de la norma jurídica es la postura de dominación; es decir, la facilidad que otorga el paradigma legislativo por no requerir en su proceso de validación de la ley un procedimiento de coherencia ni de correspondencia, tarea propia del legislador que busca sustentar los intereses de unos cuantos que no tienen relación con la protección de los derechos humanos ni de legislar para la protección de las personas." 12

Por ello consideramos que un elemento científico lo podría aportar la Teoría de la argumentación jurídica, no visto como un elemento retórico ó persuasivo que busque convencer mediante las palabras con una cierta lógica en su redacción, o en el orden que tienen de sus premisas, se le da el enfoque de elemento científico debido a la necesidad de que exista un método de verificación dentro de la ciencia jurídica, cuando existe un cambio de paradigma que tiene un objetivo distinto de estudio y sobre todo de protección a las personas.

III. Los elementos de la Teoría de la Argumentación JuRÍDICA, COMO MÉTODO CIENTÍFICO DE VERIFICACIÓN DENTRO DE LA CIENCIA JURÍDICA

\section{LA VERDAD}

Para poder llegar a tal fin como lo es la verificación dentro de la ciencia jurídica, o dicho de otra forma para poder utilizar a la argumentación jurídica como una teoría que sea considerado un método científicos dentro de la ciencia jurídica, es necesario identificar los elementos que serán necesarios para su aplicación, por ello al día de hoy consideramos como tales: verdad, lenguaje, teoría, tiempo,

12 Gonzalo Levi, Obregón Salinas., Lo Teórico y lo Practico de los Derechos Humanos., Ciudad de México, Thomson Reuters, 2018, p. 271. 
y consciencia, la suma de estos elementos o variables dentro de una conjunción nos dará la certeza e idoneidad en la ciencia jurídica.

En mi opinión, la diferencia en el uso que hoy se da a la expresión "argumentación jurídica" frente al método jurídico" radica esencialmente en que la primera tiende a centrarse en el discurso jurídico justificativo (particularmente el de los jueces), mientras que "método jurídico" (por lo menos entendido en un sentido amplio) tendría que hacer referencia también a otra serie de operaciones llevadas a cabo por los juristas profesionales y que no tienen estrictamente (o no sólo) un carácter argumentativo: por ejemplo encontrar el material con el que resolver un caso o adoptar una decisión en relación con un caso (en la medida en que se distingue de justificación esa decisión). ${ }^{13}$

La verdad dentro de la Teoría de la Argumentación Jurídica, tiene como referente que la actuación de las personas dentro de un comportamiento sea el esperado en cuanto al futuro, en el presente es lo que esta aconteciendo, y en el pasado lo que sucedió, va más relacionado con el hecho, aunado a las consecuencias que esa situación generó, una visión holística de los sucesos que permita tomar en cuenta las razones, circunstancias, para entender los resultados de esos comportamientos por parte de quien los realiza.

Los resultados en los acontecimientos (diferentes a los mencionados en una norma jurídica) son una parte importante dentro de la verdad en el comportamiento realizado por las personas para ello se debe observar las consecuencias que se generaron a partir de tal situación, para valorar si hay o no relación con lo que la norma jurídica dispone que debería de ser, verificar si existe una relación entre lo que se espera de la norma en relación con lo que acontecerá, por ello, si esperamos que los trabajadores en el caso de México tengan un número de horas máximas de trabajo, eso es lo

13 Manuel, Atienza, Luigi Ferrajoli., Jurisdicción y Argumentación en el Estado Constitucional de Derecho., Ciudad de México, UNAM-IIJ, 2017, pp. 4-5. 
que debería pasar, no que se sobre pase de ese número obligatorio de horas realizadas por los trabajadores.

En el caso de que una persona cometa homicidio, la sociedad esperaría que como consecuencia ésta deba ser privada de la libertad para su reinserción social derivado de ese comportamiento que no sólo, es contrario a la norma, además va en contra de la naturaleza de la vida, por ello, tendríamos dos supuestos el primero es que todas las personas que cometen homicidio prohibido por la legislación vayan a la reinserción social, y posteriormente a eso que las personas que realizaron esa conducta vuelvan a ser funcionales para la sociedad, para cumplir con la reinserción.

Ese es el elemento futuro e incierto de la norma jurídica, buscará que las personas en el futuro que realicen el supuesto establecido en la ley, tengan consecuencias, pero en cualquier caso de regulación tendríamos que verificar si el comportamiento se ajusta a las necesidades de la sociedad en cuanto a sus derechos humanos, de la misma manera en el caso de que se actualice el supuesto normativo, tendríamos que observar que sus fines para lo que fue creada, y las consecuencias o resultados sean los esperados.

Por esta razón es necesario que se establezca la verdad como elemento de verificación en el método de la Teoría de la Argumentación Jurídica, que la legislación a pesar de no tener un procedimiento dentro del proceso legislativo, en que valore la inclusión de la verdad como necesidad en la legislación, o que tanto los gobernantes como cualquier otra persona tenga el derecho de acceder a la verdad de los hechos, de las consecuencias, o bien que se modifique la situación que no se encuentra acorde con el momento realizado.

"La técnica - el producto de la ciencia moderna salido de la metafísica moderna - ha escapado de las manos del hombre, ha dejado de servirle, lo ha sometido y lo ha impulsado a ayudarle en la preparación de su propia pérdida. Y el hombre no conoce la salida a esta situación; no dispone de una idea de una fe y, todavía menos, de una concepción política que le permita dominar de nuevo la si- 
tuación. Impotente, contempla frío funcionamiento del mecanismo que él creo, cómo irremediablemente lo atrapa y lo arranca de sus vínculos naturaleza." 14

La verdad como elemento de la Teoría de la Argumentación Jurídica se utilizara para realizar el análisis de las premisas, los planteamientos, narraciones, dichos, se pueda verificar mediante los resultados así como las conclusiones, si la norma jurídica menciona que una persona debe vivir con dignidad, confrontar si es la falta de aplicación normativa por parte del aparato del Estado, o si la norma jurídica aunque regule tales alcances, no tiene el mecanismo para cumplir la situación de que todas las personas tengan dignidad.

Otra razón más para considerar que la verdad sea un elemento dentro de la Teoría de la Argumentación Jurídica, está relacionado con los procesos jurisdiccionales que tienen como objetivo buscar la aplicación de la norma jurídica, sin valorar que la legislación cuando es aplicada se prefiere cumplir con el formalismo antes de buscar la verdad, lo que genera una incongruencia de la ciencia jurídica que tenga como fin, por ello, tendrá que ser un elemento que sea tomado en cuenta tanto al emitir una norma jurídica como al aplicarla.

La importancia de la verdad también se relaciona con el Lenguaje, debido a que en la ciencia jurídica se utilizan símbolos y signos, relacionados a conceptos que se refieren a describir determinado acontecimiento, así como a solicitar los elementos que son necesarios para poder considerar que son los que la legislación considera como tales, por lo que le atribuirá tales efectos, por ello, el siguiente elemento que tomamos en consideración es el Lenguaje.

\section{Lenguaje}

Es un método de comunicación entre un emisor y un receptor para transmitir un mensaje, que puede ser de diferentes tipos, nos limitaremos a identificar sus elementos per se, así como la problemática

14 Václav ,Havel., El poder de los sin poder., Epublibre-prometeus, 1978, p. 154. 
que podría al momento de decodificarlo del lenguaje natural al científico, también se tiene que considerar la intensión de transmitirlo, es decir, los efectos que puede tener decir algún comentario, relacionado con lo que espero obtener.

El Lenguaje podemos considerar que es natural cuando no identifica algún elemento científico o técnico más relacionado con el comportamiento social de el día a día de las personas, consideramos lenguaje científico al que es creado para identificar y explicar tanto momentos como instituciones, y objetos específicos, conceptos creados para identificar de manera concreta mediante definición algún fenómeno o acontecimiento, de manera técnica, se podría decir que es un lenguaje creado a partir de necesidades más específicas para identificar causas y consecuencias.

"El lenguaje común es exactamente el lenguaje al alcance de todos, el lenguaje de la conversación corriente. Locke lo denomino leguaje "civil", pero quizás sea más claro hablar de lenguaje materno, ya que es el lenguaje que aprendemos en la infancia. Una vez que llega a manejar el discurso, el hombre comunica con la misma naturalidad con que respira (hasta que no está amenazado de asfixia)."15

La problemática consiste en la relación entre el lenguaje natural así como el lenguaje científico en general, ya que en determinados lugares se les puede definir de una manera distinta o bien algunas ciencias le pueden llamar de una forma u otra a un mismo fenómeno, por ello, el nivel de confusión y complejidad para las personas que no sean especialistas, o bien siéndolo no se puedan poner de acuerdo, a esto lo podemos llamar complejidad en el lenguaje científico.

"Los lenguajes especiales son los lenguajes "críticos", y mas precisamente "especializados", a lo que se llega por coerción de los defectos del lenguaje corriente. Con críticos en el sentido de

15 Giovanni, Sartori., La política, Lógica y método en las ciencias sociales., Ciudad de México., Fondo de Cultura Económica., 2000, p. 19. 
que fueron construidos mediante la reflexión sobre el instrumento lingüístico del que se valen; son especializados en el sentido de que cada disciplina tiende a crearse un lenguaje ad hoc, adaptado especialmente los problemas heurísticos que se propone." 16

El aspecto del Lenguaje como primer elemento de la Teoría de la Argumentación va relacionado con la búsqueda de reducir la complejidad del lenguaje en relación la creación de conceptos o figuras jurídicas que supuestamente pretenden dar mayor exactitud dentro de la ciencia jurídica, pero vuelven inaccesible e incomprobable la aplicación de el concepto, o bien de las consecuencias que puede tener tanto en la aplicación en una norma jurídica como dentro de un postulado científico.

Con esto se busca culminar con la complejidad en el lenguaje científico mediante la economicidad en los conceptos debido a que la ciencia debe ser creada para facilitar la compresión, aplicación, y generar mejoría para la sociedad, no debemos de llegar a considerar que para justificar una idea o postura técnica se debe buscar conceptos cada vez más complejos, lo cual sólo aleja a las personas y a la ciencia jurídica de la vida diaria.

El lenguaje dentro de la ciencia jurídica debe ser sencillo, lo más apegado al lenguaje natural en el entendimiento, para que las personas que no tienen el conocimiento relacionado con la ciencia jurídica lo puedan entender, también un concepto jurídico debe encontrarse relacionado y no estar en contra de la clasificación o definición en otras ciencias, debe encontrarse en el mismo sentido, tenemos el caso de proporcionalidad, la proporcionalidad en la ciencia jurídica se le identifica con las consecuencias relacionadas a las causas.

Aunque existe un procedimiento de razonamiento derivado de la inclusión y exclusión de las diferencias para identificar la causa y determinar las consecuencias, o bien en materia tributaria cuando

16 Giovanni, Sartori., op cit. p. 26. 
hablamos de hecho generador este debe ser proporcional a la riqueza obtenida determinando la neutralidad de la contribución de manera clásica se podría considerar que eso es proporcionalidad en las contribuciones, pero en realidad es un ejemplo de la complejidad en la ciencia jurídica en el caso mexicano.

Debido a que la proporcionalidad como se podría considerar va en la operación sencilla de cuanto obtienes frente a lo que gastas, pero en la cuestión del lenguaje científico especializado como son las contribuciones no parte de la necesidad de las personas para tener una vida digna, parte de sus ingresos lo cual es un ejemplo de la complejidad que puede llegar a ser en las ciencias en el caso de la jurídica en una especialidad como lo es las contribuciones, llegar al grado de aplicar un concepto en un contexto que parecería razonable pero que dista mucho de la realidad.

Por eso debe ser entendible y relacionado a las otras ciencias para que el lenguaje jurídico no pierde objetividad en su campo de estudio, debido a que no podemos considerar que nuestros mismos conceptos que parten de la ciencia jurídica tengan justificación debido a que están regulados en una norma jurídica, y son obligatorios lo que hace complicado que sea dudado o identificado en su contexto, las personas al contribuir al gasto público lo deben de hacer proporcional no a sus ingresos y sí a sus necesidades para obtener una vida digna.

A partir de la proporcionalidad dentro del lenguaje especializado se valora el incremento positivo al patrimonio, (se deja de lado las necesidades dignas de las personas para subsistir) lo que muestra que la palabra proporcionalidad puede ser vaga en la aplicación científica de la contribución para las personas, como se conoce en el lenguaje especializado en materia tributaria a los seres humanos se les denomina en materia de contribuciones personas físicas.

La particularidad es que las personas físicas están relacionadas con la familia y previamente se encuentra la necesidad económica de cumplir con los gastos de sostenerla, lo que distorsiona totalmen- 
te el concepto de proporcionalidad en las normas jurídicas al aplicar un concepto de proporcionalidad degradado en su aplicación, sin un referente claro que identifique el supuesto de que no deberá de contribuir al gasto público hasta que no cumpla sus necesidades más básicas.

La proporcionalidad en materia de contribuciones es un concepto vago debido a la perspectiva positivista del legislador, lo interesante se identifica cuando los teóricos parten de ideologías desde su visión de vida, o sus intereses que muestra, como en ocasiones el objetivo de la ciencia jurídica se desvía de servir al hombre para que llegue al bien común, o se pierde el estado de satisfacción a sus necesidades lo anterior por la especialización del lenguaje (es más evidente cuando se aplica el positivismo), es por ello, que consideramos el Lenguaje como elemento de la Teoría de la Argumentación Jurídica.

3. TEORÍA

Las posturas científicas dependen de la forma de clasificar e integrar los conceptos que son identificados mediante principios básicos o esenciales dentro de la ciencia jurídica, (como se analizó en el apartado anterior del principio de proporcionalidad) estos conceptos se van desarrollando mediante otras definiciones para buscar una mejor explicación de los acontecimientos, con el afán de llegar a la mejor precisión en la identificación del referente, lo que debe permitir suponer las consecuencias derivado de las causas una vez identificadas.

Las teorías de la ciencia jurídica en la actualidad parten de dos ideas o premisas de estudio la norma jurídica o la persona, si observamos la aplicación ortodoxa sin verificación estaremos hablando de una teoría positivista, si relacionamos la norma jurídica más la razón estaremos en el neopositivismo, si aplicamos las premisas o principios directos desde la constitución consideraríamos al 
neoconstitucionalismo, o bien si la realidad social la conjugamos con la los conceptos científicos comprobables que ofrezcan soluciones inmediatas a los retos de la actualidad social se identifica el pragmatismo.

Es difícil considerar cuál teoría sería la correcta para resolver un fenómeno como problemática dentro de la ciencia jurídica, la decisión no debe venir de elementos subjetivos por el gusto de aplicar la teoría, principalmente se debe tomar en cuenta la posibilidad de resolver la problemática actual, también dar los parámetros para el bienestar de la sociedad, partiendo del principio del valor de la ciencia, (para lo que fue creada), cabe precisar que el valor científico es el objetivo de la ciencia la justificación de su creación.

La teoría al homologar, y sistematizar conceptos para dar una posible respuesta al fenómeno como problema, pueden llegar a tal extremo de cumplir un objetivo distinto al que la ciencia jurídica ha creado, como ejemplo podríamos considerar la formalidad (proceso legislativo) necesaria para la creación de las normas (dejando de lado el elemento material) frente a la falta de regulación de la necesidad de las personas para vivir de una manera digna, existe como premisa principal dentro del positivismo la siguiente: no importa que la persona no goce de su dignidad, primero es la norma, si la ley considera que no viola su dignidad, entonces así es.

La complejidad de saber qué Teoría utilizar para tratar de resolver los retos que se presentan a partir de las problemáticas que se deben resolver, depende de lo accesible en su entendimiento y posible aplicación, por lo tanto antes de realizar grandes conceptos u oscuros esquemas racionales, previamente debemos preguntarnos al utilizar una Teoría, para qué sirve la información clasificada, identificada y sistematizada, además de analizar la verificación o acreditamiento desde su práctica, también debe responder a la pregunta: ¿es necesario tanta especificidad, complicación de referente o significado cuando las personas no podrían entender tales conceptos creados? 
Es importante reflexionar cuál es la importancia y objetivo en la creación de una Teoría, para entender la postura ideológica, más aun, analizar si al momento de su aplicación la Teoría cumple con el postulado científico, o solamente es lógico en el desarrollo de la Teoría sin que de manera interna exista contradicción, relacionada a su entendimiento así como practicidad para resolver la problemática.

Los expositores de la Teoría deben tener la rigurosidad científica de demostrar su aplicación en la sociedad así como sus beneficios, no solamente desde una postura doctrinaria de relación entre un concepto con otro, o lo impactante que podría ser para el auditorio la dificultad de entender definiciones mediante enunciados muy elaborados, de la misma forma basar el éxito de la Teoría en la complejidad para entenderla para nosotros esa postura solamente justifica la obscuridad de esa reflexión al no resolver la dificultad de cumplir las necesidades más básicas mediante la Teoría.

La justificación que consideramos para que la Teoría forme parte de un elemento como la argumentación jurídica como método científico de comprobación de la ciencia jurídica, es porque busca cuestionar a las Teorías y sus exponentes para que demuestren la aplicación y reducción de procesos para exigir un derecho humano, frente a particulares o al Estado cuando es transgredido y que exista la posibilidad de identificar la premisa Teórica de manera clara con la economicidad de términos o conceptos.

Mediante la Teoría como elemento podemos identificar, si los doctrinarios o exponentes al momento de formular conocimiento novedoso, éste cumple con las características de la ciencia jurídica al suplir la deficiencia de la norma jurídica, así como reducir la necesidad de regulación e identificación de todos los derechos humanos.

El neopositivismo considera a la garantía como una necesidad sine qua non, por lo tanto considerar que el legislador es el único con la obligación de garantizar hace que en México sea imposible 
la exigibilidad de los derechos humanos (tomando en cuenta que no somos uno de los países que más los respete), con ello proponer que el poder legislativo sea el único que genere las garantías para protección de las personas, y no incluir a los juzgadores para que en el ámbito de sus competencias al momento de resolver sean estos los que en sus decisiones garanticen los derechos que se solicitan mediante los hechos expuestos, solamente llevaría al retraso de protección y que no se aplique en el tiempo que se necesite debido a la espera o tardanza del legislador en realizarlo.

\section{TiEmpo}

El Tiempo en relación a la norma jurídica, es un elemento no menos importante, debido a que la ley como tecnología tiene una característica muy particular que es la atemporalidad de su aplicación, es decir para actualizar una norma jurídica las personas interesadas en su cumplimiento tienen que esperar hasta que los acontecimientos hayan sucedido.

Para identificar esta aseveración es importante observar la redacción de la mayoría de las normas jurídicas, sobre todo aquellas que buscan identificar momentos y otorgar consecuencias, estas normas se encuentran redactadas en futuro, lo que presupone que los acontecimientos redactados en la norma jurídica se podrán materializar una vez que se realicen los supuestos fácticos-jurídicos, lo que parece a simple vista muy sencillo, la complicación comienza con la verificación del supuesto entre los elementos frente a la creación del suceso o decodificación de la hipótesis jurídica, cuando este se ha convertido en pasado.

Lo que comienza a complicar la observación de este fenómeno radica en que la aplicación de la norma jurídica versa sobre situaciones o hechos que ya acontecieron, por lo tanto, hay una contingencia temporal entre la redacción a futuro de las normas jurídicas con su aplicación que vendrá una vez que los supuestos se 
hayan realizado, es decir del pasado, no podemos hablar de presente debido a que éste es instantáneo, es decir el momento presente se extingue cuando se realiza o solamente tiene esa característica cuando lo estas haciendo, razón por la cual hablamos de construir un nuevo hecho ya que el que se pretendía regular a futuro, ahora se volvió pasado, situación que genera contingencia para la aplicación de una norma jurídica.

Lo anterior identifica perfectamente que el tiempo para la tecnología normativa no tiene la misma medición que los momentos $\mathrm{u}$ horas de la vida de las personas, derivado a que no se encuentra de manera lineal en segundos, minutos, horas, días, meses, años, la norma jurídica puede estar con más de 50 años de regulación y continuar viva, que dentro de la ciencia jurídica a ese tiempo de vida le podríamos decir vigente, diferente a la vida del hombre que puede durar un número de años determinados lo que se encuentra condicionado a situaciones de salud, económicas, sociales, por mencionar algunos ejemplos.

Las normas jurídicas como tecnología son modificadas derivado de un proceso legislativo que es necesario hasta que el gobernante lo solicita o bien las fuerzas políticas están de acuerdo, sin tomar en cuenta la urgencia o necesidad de expedición de una ley, al partir de una postura positivista o neopositivista al considerar la imperiosa necesidad de una garantía o que la norma jurídica así lo establezca de manera exacta.

La perspectiva temporal de la norma jurídica lleva a considerar que existe una temporalidad distinta entre las personas y la norma, no sólo en su redacción a futuro, también en la actualización, por un lado tenemos a la regulación del supuesto jurídico-fáctico, que es el identificado en la ley, y por el otro el supuesto fáctico que es la vida de las personas, por qué podríamos considerar que es distinto el supuesto normativo jurídico-fáctico (ley), que el supuesto fáctico (persona), todo radica en la actualización de la norma, si la persona no cumple con los requisitos o elementos establecidos en la norma 
jurídica, no podríamos hablar de actualización ni mucho menos de esperar consecuencias.

El Tiempo como elemento de la Teoría de la Argumentación Jurídica, se caracteriza como el factor que permitirá identificar las ideas o las razones en momentos o acontecimientos, la diferencia temporal de realizar un trabajo o bien de resolver un pensamiento en duda, como en el campo del derecho la aplicación de los argumentos como premisas, razones para defender o sustentar derechos y obligaciones, dependerá no sólo de la persona que tenga mayor conocimiento de los elementos que se han mencionado anteriormente, también lo compone quien pueda realizar la verificación de manera más rápida con el mínimo de inexactitudes ante la contingencia de la decodificación y actualización normativa.

Esto quiere decir que el operador que pueda identificar de una manera más rápida con mayores elementos dentro de un acontecimiento, negocio, práctica jurídica podrá apropiarse de la atemporalidad de la legislación al generar un proceso de abstracción mayor que el del creador de la norma o bien dentro de su oponente en un proceso jurisdiccional o contencioso, para ello, es necesario considerar la atemporalidad de las figuras jurídicas que esperan ser actualizadas mediante el cumplimiento, no del momento de su realización, va más allá, dependiendo del operador o decodificador de los acontecimientos frente a los elementos de la norma jurídica para que ésta tenga consecuencias.

Situación que se puede aseverar del siguiente planteamiento:

Los obreros no deben utilizar sus economías cuando les parezca por ejemplo para hacer una huelga o celebrar fiestas. Surge entonces la necesidad de controlar las economías del obrero y de ahí la creación, en la década de 1820 y sobre todo a partir de los años 40 y 50, de las cajas de ahorro y las cooperativas de asistencia, etcétera, que permiten drenar economías de los obreros y controlar la manera en que son utilizados. De este modo el tiempo del obrero, no sólo el tiempo de su día laboral, sino el de su vida entera, podrá efecti- 
vamente ser utilizado de la mejor manera posible por el aparato de la producción. Y es así que a través de estas instituciones aparentemente encaminadas a brindar protección y seguridad se establece un mecanismo por el que todo el tiempo de la existencia humana es puesto a disposición de un mercado de trabajo y de las exigencias del trabajo. La primera función de estas instituciones de secuestro es la explotación de la totalidad del tiempo. Podría mostrarse, igualmente, cómo el mecanismo del consumo y la publicidad ejercen ese control general del tiempo en países desarrollados. ${ }^{17}$

Una de las complejidades de la norma jurídica como tecnología implica su actualización es decir hacer que mediante documentos, razones, abstracciones se revise si tiene o no aplicación una vez que los hechos se han suscitado, por ello, la aplicación no viene a posterior de la realización del acto, se perfecciona en el tiempo en que se reúnen los requisitos para su actualización, lo que dependerá en la reflexión de las partes dentro de un negocio jurídico, por ello, es una variable importante para la Teoría a de la Argumentación Jurídica.

El tiempo en relación a la toma de decisiones o aceptación de premisas, radica en que la consciencia aplicada al conocimiento y la velocidad de pensamiento para resolver tal postura, implica una apertura temporal diferente debido a que la reflexión de uno de las partes puede conducir el momento, es decir dirigir la ocasión o la discusión, con lo que generar un ambiente o contexto que parte de su idea o postura, la cual podría o no tener mayor tiempo reflexionando dependiendo de la velocidad de darse cuenta de las causas y consecuencias de su actuar, como en el caso que se menciono de los obreros.

El hecho de que la persona que llega a un nivel de consciencia mayor con más velocidad dentro de la norma jurídica, puede o no lograr que se actualice y su postura se tome como real y aceptable

17 Michel, Foucault., La verdad y las formas jurídicas., Barcelona, Gedisa., 2010, p. 129 . 
dentro de una negociación o discusión, o litigio, por la atemporalidad de la norma jurídica frente a la capacidad de consciencia de el emisor de la premisa, lo cual lleva la necesidad de estudiar el último elemento.

\section{CONSCIENCIA}

Este elemento esta relacionado con la reflexión, razonamiento, y abstracción que tiene que ver no sólo con el entendimiento de las ideas, también tiene que ver con el objetivo de la creación de los conceptos en la etapa de construcción de conocimiento, con su entendimiento teórico y en su aplicación, entender la funcionalidad que puede generar para la sociedad, así como las causas y las consecuencias de los supuestos normativos en relación con las teorías, la verdad, lenguaje y temporalidad.

El elemento consciencia es la aplicación de todos las variables anteriores (Verdad, Lenguaje, Teoría y Tiempo) lo que permite distinguir cuándo una teoría no cumple con su función dentro de la ciencia jurídica, por lo que partir de una Teoría que no entendemos así como la complejidad de sus conceptos a las razones de su comportamiento, nos lleva a ubicarnos dentro de la temporalidad por lo que continuar con esa forma de vida, nos llevará a vivir el tiempo de otra persona sus decisiones, deseos, o bien el estar inmerso un situaciones en las que no decidimos y que realizamos por inercia de las circunstancias al no poder distinguir una forma diferente de actuar ante lo cotidiano, como en el caso del obrero anteriormente mencionado.

La consciencia también permite dentro del lenguaje encontrar las intensiones que tiene el emisor del mensaje, ya que en algunas ocasiones se puede decir el tipo de intensión que tengo cuando digo o externo una idea, pero en otras ocasiones, no se menciona que intensiones existen de realizar un comentario o transmitir un men- 
saje, lo cual podría traer consecuencias no previstas para el receptor de el pensamiento y actuar de una manera desfavorable.

La importancia de un método de verificación dentro de la ciencia jurídica es imprescindible para concluir sobre la aplicación de la norma jurídica, o bien, para encontrar la verdad en los acontecimientos, enunciados, o posturas sobre todo en el momento histórico en el que nos encontramos que es el de cambio de paradigma en México e identificar si se pretende aplicar el paradigma de Estado Legislativo o bien el de Estado Constitucional.

La importancia de aplicar un método científico como el de la Teoría de la Argumentación Jurídica, es para verificar la verdad de las disposiciones normativas, de las teorías actuales, así como constatar el lenguaje en cuanto su definición, referente, significado, y no permitir que mediante conceptos obscuros por estar muy elaborados sin que se tenga claridad o inteligibilidad de sus alcances o referentes, podamos caer en el comportamiento equivocado sin estar conscientes de saberlo, por lo que podríamos encontrarnos en la toma de decisiones que no son nuestras y defender derechos que tampoco son los nuestros, o permitir la transgresión de nuestros derechos humanos, por no entender los alcances de un enunciado o comportamiento, o bien vivir en un tiempo que no es el nuestro.

\section{Bibliografía}

Albert, Hans., La ciencia del derecho como ciencia real., Giudad de México, fontamara, 2007

Atienza, Manuel y FeRRAJOLI, Luigi, Furisdicción y Argumentación en el Estado Constitucional de Derecho., Ciudad de México, UNAM-IIJ, 2017.

Foucault, Michel., La verdad y las formas jurídicas., Barcelona, Gedisa., 2010.

Häberle, Peter., Verdady Estado Constitucional., Ciudad de México, UNAMIIJ, 2006. 
Hart, H. L. A. , El concepto de Derecho., Buenos Aires., Abeledo Perrot., 2012.

Havel, Václav., El poder de los sin poder., Epublibre-prometeus, 1978.

Obregón Salinas, Gonzalo Levi., Lo Teórico y lo Practico de los Derechos Humanos., Ciudad de México, Thomson Reuters, 2018.

Ribero Toral, Gerardo., Verdad y Argumentación furídica., Guanajuato, Porrúa, 2009.

Tamayo y Salmorán, Rolando., El derecho y la ciencia del derecho, Introducción a la ciencia jurídica, Giudad de México, Fontamara, 2011.

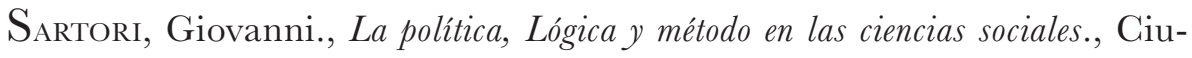
dad de México., Fondo de Cultura Económica., 2000. 